\title{
Review: Segmentation of Fovea and Optic Disc
}

\author{
Chaithra Sree. $\mathbf{K}^{1}$, K.V. Geethika ${ }^{2}$, Anil Kumar. K. R ${ }^{3}$ \\ Student, Electronics and Communication, NSS College of Engineering, Palakkad, India ${ }^{1,2}$ \\ Asst. Prof, Electronics and Communication, NSS Collegeof Engineering, Palakkad, India ${ }^{3}$
}

\begin{abstract}
Retinal image analysis is one of the crucial topics in medical image processing. During the last three decades, people are trying to extract the different features (like blood vessels, optic disk, macula, fovea etc.) automatically from retinalimage. Fovea and optic disc are the important features of a fundus retinal image.The quantitative analysis of retinal images is of increasing importance in the diagnosis of the various eye disorders. Hence a number of algorithms and techniques are being developed for its segmentation. This paper analyses various algorithms and techniques proposed earlier for the detection of optic disc and fovea.
\end{abstract}

Keywords: Optic Disc, Fovea localization, Fundus image, Segmentation, Image processing.

\section{INTRODUCTION}

Retinal image analysis is increasingly prominent as a nonintrusive diagnosis method in modern ophthalmology. The segmentation of retinal image structures has been of great interest because it could be used as a nonintrusive diagnosis in modern ophthalmology. The morphology of the fovea and the optic disk is an important structural indicator for assessing the presence and severity of retinal diseases such as diabetic retinopathy, glaucoma and age related macular degeneration. However, to assess the shape of the optic disc, manual planimetry has commonly been used by ophthalmologists, which is generally time consuming and prone to human error, especially when the vessel structures are complicated or a large number of images are acquired to be labelled by hand. Therefore, a reliable automated method for retinal blood vessel and optic disk segmentation, which preserves various vessel and optic disk characteristics, is attractive in computer-aided diagnosis.

An automated segmentation and inspection of retinal blood vessel features such as diameter, colour, and tortuosity as well as the optic disk morphology allows ophthalmologists and eye care specialists to perform mass vision screening exams for early detection of retinal diseases and treatment evaluation. This could prevent and reduce vision impairments, age-related diseases, and many cardiovascular diseases, as well as reduce the cost of the screening.

Over the past few years, several segmentation techniques have been employed for the segmentation of retinal structures such as blood vessels, fovea and optic disks in fundus retinal images. Several methods has been proposed for the localization and segmentation of optic disc and fovea. Methods like thresholding, clustering, histogram based, level set, etc. are some of them.

In this review paper a detailed survey on 25 papers on optic disc and fovea segmentation is carried out. From the detailed study that we made, the different methods of retinal image analysis, different steps involved, its merits and demerits were understood.

\section{A. Eye structure}

1) Retina :

-Nerve layer that lines the back of the eye.

-Senses light, and creates impulses that travel through the optic nerve to the brain

2) Retinal Fundus :

-Interior lining of the eyeball

-Includes the optic disc and the macula

3) Optic disc :

-Brightest part in normal fundus retinal images

-Region of entrance of blood vessels and optic nerves to the retina.

-Works as a landmark to other features in the retinal fundus image.

4) Macula :

-Small spot where vision is keenest

-At the center of the retina

5) Fovea :

-Centre most part of the macula

-Crucial for reading, watching, driving etc. 
Vol. 6, Issue 5, May 2017

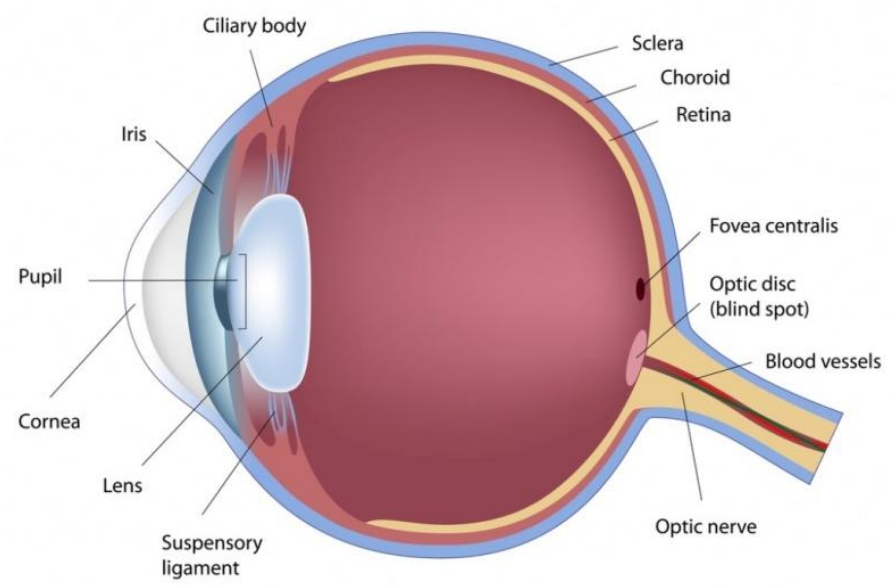

Fig 1: Human Eye Structure

(Courtesy: https://anatomyclass123.com/diagram-of-the-human-eye/diagram-of-the-human-eye-human-eye-wikipedia2/,January 26, 2017, Time: 1:57pm)

\section{REVIEW OF METHODS}

In the paper proposed by Anushikha Singh, Malay Kishore Dutta, M.Parthasarathi, RadimBurget and KamilRiha[1], a method of automatic optic disc segmentation based on region growing technique with automatic seed selection. In this method centre of optic disc is considered as a seed to apply region growing technique to segment the optic disc from the preprocessed retinal image. Automatic detection of centre of optic disc is done by double windowing method. The algorithm uses image processing techniques like contrast adjustment, morphological operations and filtering to process the retinal image and to remove the blood vessels from the retinal image. The performance of optic disc segmentation by proposed method is compared with Optic disc segmentation by ophthalmologists and results are found convincing and efficient. The experimental results indicate this method of segmentation of the OD has good accuracy and also is computationally cheap.

A fast and automated method of the macula fovea center detection in retinal images was presented by ShaohuaZheng, Lin Pan, Jian Chen, Lun Yu [2]. The approach is based on foregone anatomical structures of retina constraints on the relative locations and the intensity information within the OD (optic disc) circle boundary. Firstly, OD circle boundary is obtained accurately using adaptive thresholding, morphological bottom-hat transform and region based active contour model techniques; and then the fovea region is selected by analyzing the intensity information within the OD. Lastly, macula fovea center is detected using mathematical morphology. The public DRIVE, HEI-MED and DIARETDB1 databases are used to test the performance of the algorithm. The MF center is detected correctly in $100 \%$ of the 35 images cases in the DRIVE database, in $98.8 \%$ of 169 images cases in the HEI-MED dataset, and in $93.3 \%$ of the 89 cases in the DIARETDB1 database. It takes only about 12 seconds per image because of not relying on blood vessel segmentation in advance, which is the main advantage of the presented approach.

The paper by Xiangqian $\mathrm{Wu}$, Baisheng Dai and Wei Bu[3] presented a novel method to automatically localize OD in retinal fundus image based on directional models. In this model, the main vessels are modeled by using two parabolas with a shared vertex and different parameters. Then, to characterize the local vessel convergence in the OD as well as the shape and the brightness of the OD a local directional model, named the disc directional model, is built. Finally, the global and the local directional models are integrated to form a hybrid directional model. The proposed method was evaluated on nine publicly available databases, and achieved an accuracy of $100 \%$ for each database.

An automated fundus image analysis system was developed by Aswani S Babu[4] for the detection of optic disc, blood vessels, fovea etc. and hence the identification of differenteye diseases. The detection and analysis were carried out in three different stages namely the extraction of the candidate region (pre-processing), features extraction and classification. The fovea region is detected with the help of Advanced Adaptive Histogram Equalization, thresholding method and smoothening method. Gabor Filter and Support Vector Machine were also used for classifying the features and its similar parts. The data base was collected from LOTUS EYE HOSPITAL, Coimbatore. The proposed work has provided accuracy of 0.9022 for optic discs segmentation.

S. Shekhar and A. K. Nandi[5] proposed a novel method for the automatic segmentation and localisation of optic disc and fovea using morphological operators and the hough transform. The spatial relation from the optic disc and the 
spatial relation from the macula luteawas used to analyse fovea. Hough transform was used to localise optic disc and then macula was identified within the region of interest by iteratively applying a threshold, and then applying morphological opening. The intensity variation of macula and fovea is less compared to the variation caused by the noise and hence fovea can be easily localized. The proposed method was tested on the drive database and correctly localized the optic disc with a success rate of $94.4 \%$. The fovea was localized with a success rate of $100 \%$.

Aliaa Abdel-Haleim Abdel-RazikYoussif, AtefZakiGhalwash, and Amr Ahmed Sabry Abdel-RahmanGhoneim [6] in their proposed a method for the detection of optic disc which is an important step for the identification of diabetic retinopathy.At first, luminosity and contrast throughout the image will be normalizing using illumination equalization and adaptive histogram equalization methods respectively. Matching of the expected directional pattern of the retinal blood vessels is the base of the algorithm used for the detection of OD. Hence, to match the vessels direction at the OD vicinity a simple matched filter is proposed. The 81 fundus images of the STARE project's dataset which contains both normal and diseased retinas was used to evaluate the proposed method.80 out of the 81 images (98.77\%) was correctly detected for the OD-center. The uneven crescent shaped illumination near the border biased the OD candidates thus affecting the vessel segmentation algorithm which in turn can lead to the incorrect detection of OD. Also using the publicly available DRIVE dataset, the OD-center was detected correctly in all of the 40 images (100\%).

Thomas Walter, Jean-Claude Klein, Pascale Massin, and Ali Erginay[7] proposed a method for the identification of exudates within the macula is an important task in the detection of diabetic macular edema. Using their high grey level variation, exudates are found. Morphological reconstruction techniques are used for the determination of the exudates counters. In this approach the detection of the optic disc is indispensable. Morphological filtering techniques and the watershed transformation are used for the detection of optic disc. Small image database was used for the testing of algorithm and then compared it with the

performance of a human grader. A sensitivity of $92.8 \%$ and a mean predictive value of $92.4 \%$ was obtained. Three different ways in which the image processing of color fundus images contribute to it are image enhancement, mass screening (including detection of pathologies and retinal features), and monitoring (including feature detection and registration of retinal images). By changing various parameters, the robustness of the algorithm was evaluated.

Here Muhammad Abdullah, Muhammad MoazamFraz and Sarah A. Barman [8] have proposed the method based on morphological operations, the circular Hough transform and the grow cut algorithm. For the enhancement of the optic disc and for the removal of the retinal vasculature and other pathologies, morphological operators are used. Circular Hough transform and the grow cut algorithm are employed to approximate the optic disc and precisely segment the optic disc boundary respectively. Retinal images for the evaluation of the proposed method are from five publicly available databases DRIVE, DIARETDB1, CHASE DB1, DRIONS DB, MESSIDOR and one local Shifa Hospital Database. Except DRIONS DB, MESSIDOR, and ONHSD databases, the proposed method achieved an optic disc detection success rate of $100 \%$. For DRIONS-DB, MESSIDOR, and ONHSD databases the method achieved a success rate of $99.09 \%$ and $99.25 \%$. Through the experimental evaluation, it shows that the grow-cut algorithm is computationally fast in processing, robust to the variations in image contrast and illumination, works well in pathological retinal images and is comparable with state-of-the-art methodologies in terms of quantitative performance metrics. During the evaluation process using the three types of generally found biomedical noises, Gaussian, Salt \& pepper, and Specklenoise, the retinal images have been corrupted. Despite of the significant deterioration of the retinal images, the methodology successfully segmented the OD.Based on its OD localization and segmentation performance on the noisy images, the images with illumination artefacts, and the images with pathological structures, the robustness of proposed methodology has been evaluated. This method has shown significant improvement over existing methods at that time.

In the study of authors Cananelik, PakizeErdomu,[9] they proposed a method for optic disc detection, which is the first step in diagnosing many eye related diseases. Here the blood vessels in the retinal images, obtained from the MESSIDOR are eliminated by using red channel. For obtaining the optic disc Graph Cut algorithm is implemented on the retinal images by eliminating the noise operations. Due to its results in specially vessel segmentation, recently this algorithm gained importance. In terms of the originality of the algorithm used in this study and the success rate achieved, the results are encouraging

In a study, D.JudeHemanth, J.Anitha [10] proposed a novel approach for the segmentation and feature extraction of OD is emphasized. For the segmentation they have used a hybrid method involving fuzzy and level set method. Herethey have tried to explore the hidden advantages of several segmentation techniques. By extracting few shape based features from the segmented anatomical structures, validation of this technique was performed. It is difficult to compare it with other works because its results are given qualitatively. Real time application of this method is also explored in this research. 
A new approach has been proposed by Ana SalazarGonzalez, DjibrilKaba, Yongmin Li, and Xiaohui Liu [11] for the extraction of the retina vascular tree is using the graph cut technique. Location of the optic disc is estimated using the blood vessel information. It is done using two alternative methods, Markov random field (MRF) image reconstruction method and compensation factor method. In MRF method optic disc is segmented by removing vessels from the OD region. And in compensation method optic disc is segmented using the prior local intensity knowledge of the vessels. The method is quantitatively evaluated using three public datasets, DIARETDB1, DRIVE, and STARE. Both on the DIARETDB1 and DRIVE images, MRF image reconstruction algorithm outperforms the compensation factor algorithm by $2.56 \%$ and $11.5 \%$, respectively. But the performance of the MRF image reconstruction algorithm depends on thevessel segmentation algorithm. The overlapping tissue segmentation is an important issue faced by nowadays medical analysis, which the proposed method addresses. So, in other medical image analysis applications to overcome the overlapping tissue segmentation this method can be applied.

The authors DharitriDeka, JyotiPrakashMedhi, S. R. Nirmala [12] proposed a new approach known as DWT(Discrete Wavelet Transform) for the detection of macula and fovea through this paper. Macula which is responsible for central vision is located nearly in the center of the retina. The closely packed cone cells in the center of the macula is called the fovea. Macula may get affected due to different diseases like Diabetic Retinopathy (DR), Diabetic Macular Edema (DME) and Age-related Macular Degeneration (AMD) etc. Therefore early detection of these diseases is necessary. Hence for the analysis of these diseases detection of macula and fovea plays an important role. Localization of macula is done through the investigation of the blood vessels in the macula region. Validation of the proposed method is done by testing 1020 numbers of images from the databases DRIVE, DIARETDB1, STARE, MESSIDOR and HRF. Test image accuracies are 100\% (36 36), 97.75\% (782 800), 95.5\% (85 89), 96\% (48 50), 100\% (45 45) for DRIVE, MESSIDOR, DIARETDB1, STARE, HRF respectively. And in total 97.85\% (996-1020). Those images that have poor illumination and thus effected the fovea detection were eliminated.

Veena Raj and Dr.Vidya Devi [13] introduced an unremarkable method for the detection of fovea from noisy images. Images taken for the validation are exposed to two types of noises, Salt and pepper noise and Gaussian noise. Adaptive wavelet filter and Modified Decision Based Unsymmetrical Trimmed Median Filter (MDBUTMF) were used for denoising the images. Based on PSNR values the efficiencies of both the methods were compared. And then blood vessels and fovea were detected using Mathematical Morphology. Its validation was done using DRIVE database.

Rodrigo Veras, Romuere Silva, Kelson Aires, Fatima Medeiros [14] introduced a new method for fovea detection in colour retinal images by combining green and red channels. They proposed an algorithm using pseudo-code to detect the fovea center on the input images. Evaluation of images were carried on three public benchmark databases, DRIVE , ARIA ( $100 \%$ of success rate) and MESSIDOR. Segmentation algorithm searches for the lowest mean colour intensity window that results from a fusion between the red and green channels. Identified that, the algorithm design and colour channels have a dependency relationship among the structures to be detected.

In the paper by Michael D. Abramoff, MeindertNiemeijer, Bram van Ginneken [15], a method to detect the approximate position of the optic disc using $\mathrm{kNN}$ regression is presented. In this method a regression model of the optic disc position is build initially. Using a prior vessel segmentation all vessel pixels are searched for those which are inside the optic disc according to the regression model. To handle noise, the regression output is blurred. The point which is closest to the middle of the optic disc is chosen. The method was tested on 1000 screening images and was able to find the correct position in $99.9 \%$ of all cases.

SangitaDhondiba Bharkad [16] proposed a new method for the automatic segmentation of the Optic Disk (OD) in retinal images. The proposed OD segmentation method is based on Discrete Wavelet Transform (DWT) and morphological operations. This method was evaluated on publicly available standard databases DIARETDB0 and DIARETDB1. The proposed method attained accuracy rate $89.88 \%$ on DIARETDB0 and $91.53 \%$ on DIARETDB1 database for localization of the OD. Average sensitivity and specificity for this method was found to be $71.54 \%$, $99.53 \%$ and $71.11 \%, 99.56 \%$ on DIARETDB0 and DIARETDB1 databases respectively.

Sanjeev and Mila [17] proposed novel method for automatic segmentation of optic disc using a modified multithresholding technique on a preprocessed image. After sufficient preprocessing on the fundus image, segmentation was done using multi level thresholding. The segmented results was extracted using morphological operators. The result obtained was then compared with those obtained using $\mathrm{K}$ means cluster method. The algorithm was tested over 50 images and accuracy of $94 \%$ was obtained.

The paper by SumanSedai et al.[18] provides a method for the accurate segmentation of optic disc in retinal colour fundus images using an automatic regression based method. The approximated optic disc obtained after segmenting 
using circular hough transform is used to compute the initial cup shape. The method of regression analysis is a statistical process for estimating the relationships among variables and the cascaded shape regression method iteratively learns the final shape of the optic cup and disc from an initial shape. Gradient boosted regression trees are employed to learn each regressor in the cascade. The proposed method provided high segmentation accuracy for optic cup and disc segmentation with dice metric of 0.95 and 0.85 .

Huiqi Li and Opas Chutatape[19] proposed a novel method to detect the optic disc. In this method principal component analysis is used for localization of Optic disc. A modified active shape model (ASM) is proposed to detect the disc boundary. The proposed method has achieved success rates of $99 \%, 94 \%$, and $100 \%$ for disc localization, disc boundary detection, and fovea localization respectively. The image for analysis was obtained from the Singapore National Eye Center (SNEC).

Soumitra Samanta, Sanjoy Kumar Saha and BhabatoshChanda[20] presented a simple and fast algorithm using Mathematical Morphology to find the fovea region. The proposed algorithm is based on the structure of the blood vessels and some information of the optic disc. They have tested the algorithm on a publicly available DRIVE database and have obtained comparable results with a state of the art in this area. The success rate was found to be $97.14 \%$. They have also tested on the images of their own database containing 20 images and have achieved $100 \%$ success.

The level set method proposed by Chuang Wang and DjibrilKaba [21] is the segmentation of an image by solving a partial differential equation. After the localization of optic disc, the blood vessel is extracted to reset the centre followed by level set method, which incorporates edge term, distance-regularization term and shape-prior term, to segment the shape of the optic disc. Seven measures are used to evaluate the performance of the methods. The effectiveness of the proposed method is evaluated against alternative methods on three public data sets drive, diaretdb1 and diaretdb0. The advantages is that it is implicit, parameter-free, provides a direct way to estimate the geometric properties of the evolving structure, allows for change of topology, and is intrinsic. The method provided an average sensitivity of $94.65 \%$, average specificity of $98.89 \%$, average predictive value of $93.95 \%$ over DIARETDB0 dataset and average sensitivity of $93.24 \%$, average specificity of $98.94 \%$, average predictive value of $94.23 \%$ over DIARETDB1 dataset and average sensitivity of $92.58 \%$, average specificity of $99.26 \%$, average predictive value of 95.19\% over DRIVE dataset.

Vasanthi and group [22] proposed a model that presents an automated glaucoma detection method known as colour fundus imaging (CFI) and gradient vector flow (GVF) to extract OD boundary. To overcome the problem of over segmentation, Chan-Vese (C-V) model is proposed including local image information. The second is to localize OD region and define region of interest for further processing. The contour initialization is done through circular Hough transform. To avoid the possible noise, Gaussian nose filter is used and then disc boundary detection is used through gradient vector flow model whose performance relies on contour initialization. The comparison results showed that the method is more robust and accurate than the other model, particularly in the cases of atrophy.

Shobhana.M and S.B.Chitrapreyanka[23] proposed a simple, fast algorithm using mathematical morphology to find the fovea region. The localization of optic disc is important for two purposes. The simulation tool used for processing input colour fundus images is MATLAB.

A fully automated, fast method to detect the fovea and the optic disc in digital colour photographs of the retina was presented by MeindertNiemeijer, Michael D. Abrmoff, Bram van Ginneken[24]. A kNN regressor is utilized to predict the distance in pixels in the image to the object of interest at any given location in the image based on a set of features measured at that location. The method combines cues measured directly in the image with cues derived from a segmentation of the retinal vasculature. An extensive evaluation was done on 500 images from a diabetic retinopathy screening program and 100 specially selected images containing gross abnormalities. The method found the optic disc in $99.4 \%$ and the fovea in $96.8 \%$ of regular screening images and for the images with abnormalities these numbers were $93.0 \%$ and $89.0 \%$ respectively.

The paper by AmandeepKaur and Reecha Sharma [25] discusses the automatic segmentation of the optic disc from a fundus image. For the detection of the optic disc, its approximate position is found. Then the exact contours are found by means of the watershed transformation. This method for the extraction of the optic disc contour is mainly based on mathematical morphology along with thresholding and watershed transform.

\section{III.CONCLUSION}

Segmentation is the process of partitioning an image into set of components. Segmentation of fovea and optic disc is necessary for early detection of affected area in the retina.Careful evaluation of eye structure and its documentation is 
extremely important for diagnosis and monitoring of various eye disorders. Different approaches have been developed for fovea and optic disc detection; few of the approaches are discussed in this paper.We focused on automatic segmentation of Optic disc and fovea from fundus images, which is an important parameter for the disease diagnosis. We investigated and compared performance of different methods used for Optic disc and fovea segmentation.

\section{ACKNOWLEDGMENT}

We are immensely grateful to all those who provided insight and expertise to complete our work. Also we appreciate the authors of the reference documents that turned out to be a source of resources that helped us improve our study.

\section{REFERENCES}

[1] Anushikha Singh; Malay Kishore Dutta; M. Parthasarathi; Radim Burget; KamilRiha, An efficient automatic method of Optic disc segmentation using regiongrowing technique in retinal images, 2014 International Conference on Contemporary Computing and Informatics (IC3I),IEEE, Year: 2014,PP: 480 - 484, DOI: 10.1109/IC3I.2014.7019713.

[2] ShaohuaZheng, Lin Pan, Jian Chen, Lun Yu, Automatic and Efficient Detection of the Fovea Center in Retinal images, 2014 7th International Conference on BioMedical Engineering and Informatics (BMEI 2014), DOI: 10.1109/BMEI.2014.7002760.

[3] Xiangqian Wu, Baisheng Dai And Wei Bu,Optic Disc Localization UsingDirectional Models IEEE Transactions On Image Processing, Vol. 25, No. 9, September 20164433.

[4] Aswani S Babu, Fovea Detection And Disease Identification Using IntegreatedGf-Svm Method, International Journal Of Current Medical And Pharmaceutical Research, 1, (2), 28-34, 2015.

[5] S.Sekhar, W. Al-Nuaimy ,A. K. Nandi, Automated localisation of optic disk and fovea in retinal fundus images, 2008 16th European Signal Processing Conference, Year: 2008, PP: 1-5.

[6] AliaaAbdel-HaleimAbdel-RazikYoussif,AtefZakiGhalwash, andAmr Ahmed Sabry Abdel-RahmanGhoneim,Optic Disc Detection From Normalized Digital Fundus Images by Means of a Vessels Direction Matched Filter,IEEE TRANSACTIONS ON MEDICAL IMAGING, VOL. 27, NO. 1, JANUARY 2008.

[7] Thomas Walter, Jean-Claude Klein, Pascale Massin, and Ali Erginay, A Contribution of Image Processing to the Diagnosis of Diabetic Retinopathy-Detection of Exudates in Color Fundus Images of the Human Retina, IEEE TRANSACTIONS ON MEDICAL IMAGING, VOL. 21, NO. 10, OCTOBER 2002.

[8] MuhammadAbdullah, MuhammadMoazamFraz and SarahA.Barman, Localization and segmentation of optic disc in retinal images using circular Hough transform and grow-cut algorithm, PeerJ4:e2003;DOI10.7717/peerj.2003.

[9] Cananelik, PakizeErdomu, A New Approach in Human Retina Optic Disc Segmentation using Graph Cut, 978-1-5090-1679-2/16/\$ 31.00 2016 IEEE.

[10] D.JudeHemanth, J.Anitha, Hybrid clustering method for optic disc segmentation and feature extraction in retinal images, 978-1-467348058/12/\$31.00 c 2012 IEEE.

[11] Ana Salazar-Gonzalez, DjibrilKaba, Yongmin Li, and Xiaohui Liu, Segmentation of the Blood Vessels and Optic Disk in Retinal Images, IEEE JOURNAL OF BIOMEDICAL AND HEALTH INFORMATICS, VOL. 18, NO. 6, NOVEMBER 2014.

[12] DharitriDeka, JyotiPrakashMedhi, S. R. Nirmala, Detection of Macula and Fovea for Disease Analysis in Color Fundus Images, 2015 IEEE 2nd International Conference on Recent Trends in Information Systems (ReTIS).

[13] Veena Raj, Dr.Vidya Devi, Retinal Image Analysis Using Fovea Detection using Unsymmetrical Trimmed Median Filter (MDBUTMF), International Journal of Engineering and Innovative Technology (IJEIT) Volume 3, Issue 3, September 2013.

[14] Rodrigo Veras, Romuere Silva, Kelson Aires, Fatima Medeiros, Automatic detection of fovea in retinal images using fusion of color bands, 2014 27th SIBGRAPI Conference on Graphics, Patterns and Images, 15301834/14 \$31.00 2014 IEEE DOI 10.1109/SIBGRAPI.2014.17 ,PP 119124 .

[15] MeindertNiemeijer, Michael D. Abrmoff, Bram van Ginneken, Fast detection of the optic disc and fovea in color fundus photographs, https://www.ncbi.nlm.nih.gov/pmc/articles/PMC2783621/, Med Image Anal. 2009 Dec; 13(6): 859870. Published online 2009 Sep 4. doi: 10.1016/j.media.2009.08.003.

[16] SangitaDhondiba Bharkad, Automatic Segmentation of Optic Disk in Retinal Images Using DWT, 2016 IEEE 6th International Conference on Advanced Computing (IACC), Year: 2016, PP: 386 - 391, DOI: 10.1109/IACC.2016.78.

[17] Mila Kankanala, SanjeevKubakaddi, Automatic Segmentation of Optic Disc using Modified Multi-level Thresholding.

[18] SumanSedai, Pallab K. Roy, DwarikanathMahapatra, Rahil Garnavi,Segmentation of Optic Disc and Optic Cup inRetinal Fundus Images using Shape Regression, 2016 38th Annual International Conference of the IEEE Engineering in Medicine and Biology Society (EMBC)PP: 3260 3264, DOI: 10.1109/EMBC.2016.7591424.

[19]SumanSedai, Pallab K. Roy, DwarikanathMahapatra, Rahil Garnavi, Segmentation of Optic Disc and Optic Cup inRetinal Fundus Images using Shape Regression, 2016 38th Annual International Conference of the IEEE Engineering in Medicine and Biology Society (EMBC)PP: 3260 3264, DOI: 10.1109/EMBC.2016.7591424.

[20] Soumitra Samanta, Sanjoy Kumar SahaAndBhabatoshChanda, A Simple And Fast Algorithm To Detect The Fovea Region In Fundus Retinal Image,2011 Second International Conference On Emerging Applications Of Information Technology.

[21] Chuang Wang, DjibrilKaba, Yongmin Li, Level Set Segmentation of Optic Discs from Retinal Images, Journal of Medical and Bioengineering, Vol. 4, No. 3,PP 213-220, June 2015. Doi: 10.12720/jomb.4.3.213-220.

[22] S.VASANTHI, R.ANAND, G.ARAVINTH BABU, K.DEEPA, V.LEKHA, SEGMENTATION OF OPTIC DISC IN FUNDUS IMAGES, Indian Journal of Computer Science and Engineering (IJCSE), ISSN : 0976-5166, Vol. 3 No. 2 Apr-May 2012,PP 230-234.

[23] A Shobhana.M and S.B.Chitrapreyanka, A Novel Approach For Abnormality Detection Of Macula And Fovea Region From Color Fundus Images International Journal of Emerging Trends in Electrical and Electronics Vol. 3, Issue. 2, May-2013.

[24] Michael D. Abr'AmoffAndMeindertNiemeijer The Automatic Detection Of The Optic Disc Location In Retinal Images Using Optic Disc Location Regression Proceedings Of The 28th IEEE EMBS Annual International Conference New York City, USA, Aug 30-Sept 3, 2006,14244-0033-3/06 2006 IEEE.

[25] AmandeepKaur, Reecha Sharma, Optic Disc Segmentation Based On Watershed Transform, Volume 4, Issue 5, May 2014, ISSN: 2277 128x International Journal Of Advanced Research In Computer Science And Software Engineering. 